Royal College of PSYchiatrists (1998) Management of Imminent Violence. Clinical Practice Guidelines to Support Mental Health Services (OP 41). London: Royal College of Psychiatrists.

SUGARMAN, P. \& MOSS, J. (1994) The rights of voluntary patients in hospital. Psychiatric Bulletin. 18. 269-271.

Thomas, C.. BARTLETT. A. \& MEzeY, G. C. (1995) The extent and effects of violence among psychiatric in-patients. Psychiatric Bulletin, 19, 600-604.

WOLPE, P. R., SCHWARTZ. S. L. \& SANDFORD, B. (1991) Psychiatric in-patients' knowledge of their rights. Hospital and Community Psychiatry, 42, 1168-1169.
Zigmond, A. (1995) Special care wards: are they special? Psychiatric Bulletin. 19. 310-312.

*Stephen Pereira, Consultant Psychiatrist, Dominic Beer, Consultant Psychiatrist, and Carol Paton, Principal Pharmacist, Oxleas NHS Trust, Bexley Hospital, Old Bexley Lane, Bexley. Kent DA5 2BW

*Correspondence

\title{
Open all hours: extending the role of the psychiatric day hospital
}

\author{
Judy Harrison, Amanda Poynton, John Marshall, Richard Gater and \\ Francis Creed
}

\begin{abstract}
Aims and method From March 1997, the acute day hospital in Central Manchester was extended to 24 hours, seven days a week, with patients treated entirely at home if preferred. The development and organisation of the new service are described. Preliminary evaluation data include service activity for the first 12 months and comparison with a consecutive series of in-patients during the first three months.

Results There was an increase in the number of patients treated in the first year $(n=214)$. Sixty-two per cent of patients suffered from schizophrenia. psychotic depression or bipolar disorder. Patients treated by the new service in the first three months $(n=43)$ were more likely than in-patients $(n=37)$ to have problems eating or drinking and to present with suicide risk, whereas the inpatients were more likely to have had adverse past experiences of services and to show behavioural disturbances.
\end{abstract}

Clinical implications The findings support the continued development of 24-hour alternatives to inpatient care, while emphasising that those requiring inpatient care have different presentations and needs.

\section{The original Central Manchester day hospital}

The Central Manchester day hospital was established in 1985, with a focus on acute treatment as an alternative to in-patient care. Early research on the use of the day hospital suggested that it was able to treat many patients who were as ill as those admitted to in-patient care (Creed et al, 1989). Subsequent randomised controlled trials of day hospital versus in-patient care showed that up to $40 \%$ of people presenting for admission could be treated successfully in the day hospital. with few differences in clinical or social outcome (Creed et al, 1990), and that the costs associated with day hospital care were significantly less than those for in-patient care (Creed et al, 1997).

Although the day hospital had successfully maintained a focus on acute treatment for over 10 years, staff were aware of a number of limitations. The service only operated from $9 \mathrm{am}$ to $5 \mathrm{pm}$ Monday to Fridays, leaving patients unsupported outside these hours. The addition of a limited weekend service during the second randomised controlled trial appeared to increase the severity of illness that could be treated successfully (further details available from the authors upon request). Although transport was provided and some home visits were offered, patients had to attend the base for at least some of their treatment and it was not possible to engage patients who preferred not to do so. There were also conflicting demands on the resources of the day hospital, such as group 
work, work with less severely ill patients and inpatients attending for activities.

\section{Establishment of Home Option Service}

A successful bid for an additional $\$ 235000$ per annum was submitted to the Mental Health Challenge Fund to extend the service offered. Following a six-month development period, the new service opened in March 1997, with a change in name to the Home Options Service. The manager of the new service (J.M.) was appointed in advance of any other changes, allowing him to lead the change process, and a multi-agency steering group was established with wide representation. The original day hospital had a body of very experienced staff, all of whom have continued to work in the new service. complemented by the appointment of new staff, many of whom had worked previously on the in-patient unit. All staff received training in risk assessment and psycho-social interventions, and ongoing training needs are reviewed through clinical supervision. Staff met regularly before the opening of the new service to develop policies and procedures, culminating in a two-day team-building session immediately prior to opening. The staffing of the new service consists of two charge nurses, eight staff nurses, three unqualified support workers, three occupational therapists, one occupational therapy technician plus a full-time manager and an administrative clerk. The original day hospital had medical time specifically dedicated to the service and this has been maintained with approximately four consultant sessions, four sessions of Senior Registrar time and five sessions of Senior House Officer time.

\section{Admission criteria and referral process}

The new service operates 24 hours a day, seven days a week. The team base is staffed from 9 am to $11 \mathrm{pm}$, with two members of staff on call at night. The service offers 30 places to patients aged 16-65 years who are experiencing an episode of acute mental illness that would otherwise require in-patient admission. The degree of risk to self or others must be considered manageable, and patients must be willing to cooperate with at least some aspects of the treatment package. Referrals are accepted from the in-patient unit if patients are still acutely ill but could be more appropriately cared for by the Home Options Service. Only emergency referrals are accepted, with no waiting list in operation.

Referrals to the service can be made at any time over the 24-hour period by psychiatrists, or by community-based keyworkers for service users known to the service and registered on the Care Programme Approach. For each shift, a designated team member receives and assesses new referrals and can be contacted via a bleep. The initial assessment focuses on identification of risk, compliance with the service, support networks and home circumstances. Wherever possible, carers are included in this process. Once a patient is accepted, treatment begins immediately, including taking patients home and giving medication if indicated. Patients who are not accepted remain the responsibility of the referrer, who will have to decide on an alternative treatment strategy.

\section{Treatments offered}

The frequency of contact is agreed on admission and reviewed at least weekly. Most patients are seen between one and three times a day, depending on the level of risk and the need to supervise medication. The service model combines aspects of acute day hospital and home treatment: patients are encouraged to attend the base for at least some aspects of their treatment but can be seen entirely in their own homes if preferred. Most patients are seen both at home and at the team base. If necessary, patients can be monitored on a one-to-one basis at the team base from 9 am to $10 \mathrm{pm}$ and then taken home with night-time medication. Patients and carers are given the emergency contact number on admission and can contact a member of staff at any time.

Patients are allocated keyworkers and coworkers on admission and the team is subdivided into two sector and corresponding consultant teams relating to the community mental health teams. Team meetings involving medical staff and keyworkers are held weekly. The initial focus of treatment is on engagement. reduction of symptoms and safety. Once these have been established, more information is gathered about psychosocial needs and a multidisciplinary care plan is generated to address immediate and longer term needs.

A limited range of group activities is also available at the team base, including arts-based activities, anxiety management and a support group. Wherever possible, staff help patients to access existing activities in the community. which can continue after discharge. If patients are already known to the community teams, their existing keyworker is expected to continue to work with them during the admission.

The Home Options Service also has exclusive use of a single respite bed located in a selfcontained flat within the department's hostel ward. This can be used for a maximum of one to two nights for patients who need closer supervision for a limited time period. If the respite bed is used, the staffing is provided from within the team resources. 


\section{Service activity}

During the first 12 months of the service 349 patients were referred, of whom $214(61 \%)$ were accepted for treatment. This is more than double the number treated in the old day hospital in the 12 months prior to the change in service. The most common reasons for non-acceptance were unwillingness to cooperate $(28 ; 21 \%)$, too unwell $(31 ; 23 \%)$ or not ill enough $(32 ; 24 \%)$. Thirty-four patients $(16 \%$ of those accepted) subsequently had to be transferred to in-patient care. The commonest source of referral was the accident and emergency department (140; 40\%), followed by the out-patient department $(86 ; 25 \%)$, the walk-in day-time reception area $(50 ; 14 \%)$, the in-patient unit $(27 ; 8 \%)$ and the community teams $(20 ; 6 \%)$.

Two hundred of the 214 patients admitted were assigned an ICD-10 diagnosis (World Health Organization, 1992) by the treating doctor. Of these, $80(40 \%)$ suffered from schizophrenia or a related psychotic illness, 57 (29\%) from depression, of whom 13 had psychotic depression (7\% of total), 29 (15\%) from bipolar affective disorder, 22 (11\%) from anxiety or adjustment disorders and the remaining 12 (6\%) from other disorders. Forty-nine per cent of all contacts with patients in the first 12 months took place at the service base, $42 \%$ in patients' homes and the remaining $9 \%$ at other locations.

\section{Comparison with in-patient sample}

During the first 10 weeks of the new service, details of consecutive admissions to Home Options ( $n=43)$ and to the in-patient unit ( $n=37)$ were collected. Staff members were asked to rate all patients using a modified version of the Social Behaviour Schedule (SBS; Wykes \& Sturt, 1986). Socio-demographic details, plus details of previous contact with services, were derived from the case notes. Length of admission was calculated retrospectively after discharge.

Comparing the Home Options sample with the in-patient sample, there were no significant differences in socio-demographic characteristics (46\% male $v$. 58\%; mean age 35.6 years $v$. 35.5 years; $30 \%$ from ethnic minority groups $v .37 \%$;
$39 \%$ living alone $v .44 \%$ ). The in-patient sample were more likely to have adverse past experiences of services, including being more likely to have had previous compulsory admissions, admissions in the last two years, admissions of longer than six months at some point and a history of homelessness or previous custodial sentence (Table 1). Of the 23 items in the SBS, 16 showed no difference between the in-patients and the Home Options patients. The in-patients were more likely to be rated as demonstrating attention-seeking behaviour, bizarre speech content, incoherent speech, socially unacceptable habits and overactivity, whereas the Home Options patients were more likely than the inpatient sample to be rated as having problems with eating and drinking or to pose a suicide risk. There was a tendency for the total SBS scores to be higher for the in-patient group, but the difference was not statistically significant (Table 2). The median length of stay for in-patients was 47 days (interquartile range 12-62) compared with 20 days for the Home Options sample (interquartile range 12-27) (Mann-Whitney $U$ test, $P=0.06$ ).

\section{Potential benefits of Home Options}

The service is accessible and flexible, with staff contactable at all times - an aspect of services that users rate as being highly desirable (Wood, 1994). We have treated a number of patients who have previously refused hospital admission when unwell and would have faced detention under the Mental Health Act if Home Options had not been available. Treating patients in their own homes avoids the stigma of hospital admission, prevents the breakdown of important social networks and avoids disruption to patients' benefits. For patients from ethnic minorities, the experience of hospital admission can be particularly distressing and Home Options offers an alternative treatment mode that does not disrupt cultural traditions. Contact with patients at home enables a more accurate assessment of needs, including those of carers, and patients have more intensive contact with their keyworker, allowing a more individualised care plan to be formulated. A detailed cost-benefit evaluation is needed to

Table 1. Service history of Home Options and in-patient sample

\begin{tabular}{lccc}
\hline & In-patients $(n=37)$ & Home Options (n=43) & $P\left(x^{2}\right.$ test) \\
\hline Previous compulsory admissions & $20(65 \%)$ & $10(24 \%)$ & $<0.001$ \\
Previously in hospital for more than six months on & $6(19 \%)$ & $2(5 \%)$ & 0.05 \\
one admission & $15(62 \%)$ & $12(34 \%)$ & 0.033 \\
Any admissions in last two years & $10(33 \%)$ & $2(5 \%)$ & 0.001 \\
Ever homeless & $11(34 \%)$ & $7(16 \%)$ & 0.069 \\
Previous periods in custody & & & \\
\hline
\end{tabular}


Table 2. Scores on Social Behaviour Schedule (SBS)

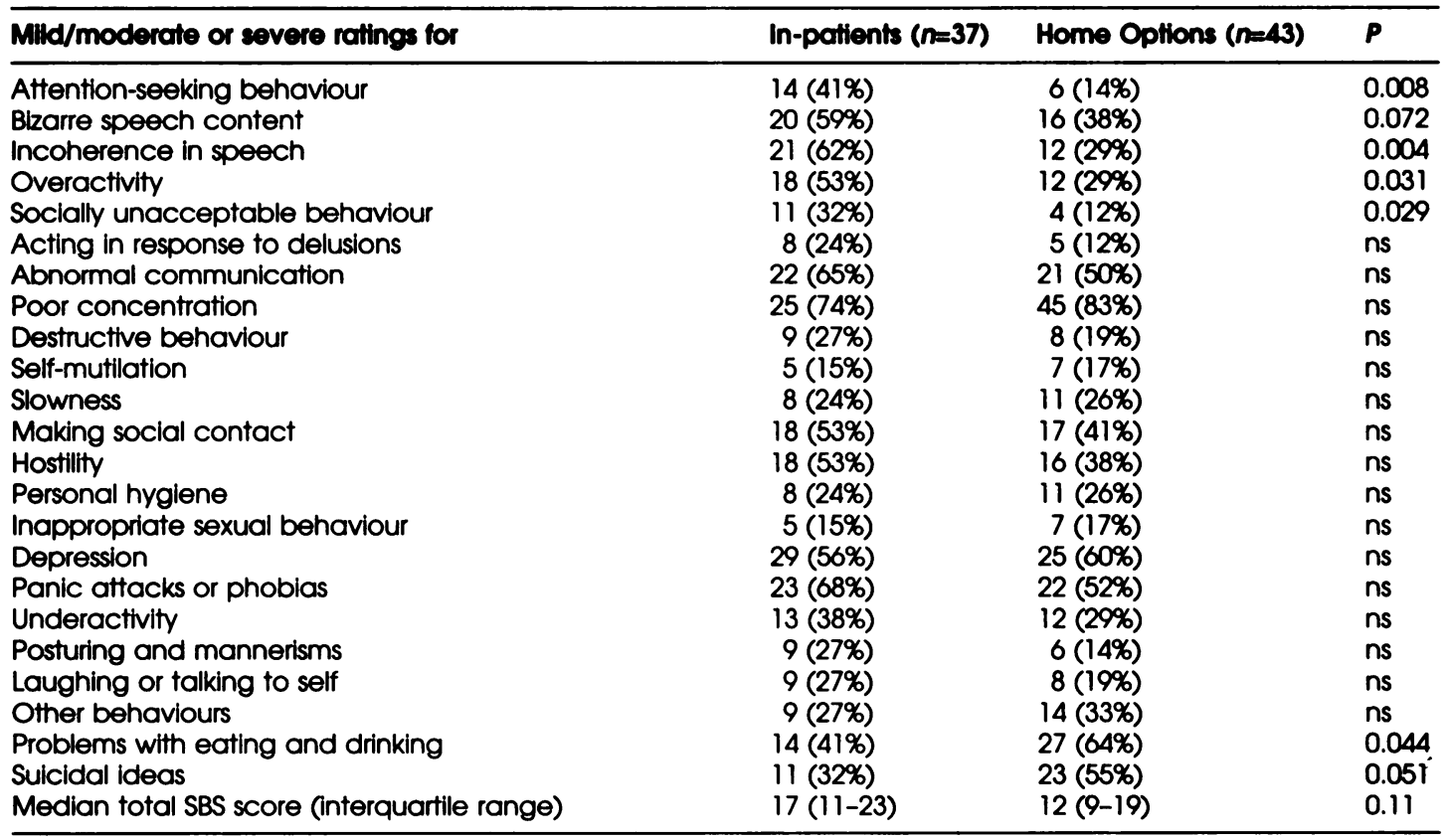

examine the costs associated with the new service, but the very high costs associated with in-patient care make it unlikely that Home Options will be a more expensive option.

\section{Potential disadvantages of the model}

Treatment at home is potentially more stressful for carers than hospital admission, although existing research does not suggest an increase in burden for carers associated with day hospital (Creed et al, 1997) or home treatment (Marks et al, 1994). Patients may also miss the 'asylum' function of an in-patient unit.

Some patients can be maintained at home, with daily contact and supervision of medication, but they relapse as soon as this intensity of service is withdrawn. A network of other community services that can provide intensive support in the longer term is needed, in addition to an acute service of this kind. This includes a need for long-term structured day care and services for patients who have disabling longterm conditions but would not be eligible for the Home Options service because their problems are not sufficiently acute. This group might traditionally have received day hospital care and are at risk of losing access to services with an increasing focus on acute treatment.

As 24-hour alternatives to in-patient care develop, the remaining patients treated in in- patient units are likely to be more difficult to treat, with increased length of stay and greater mean costs, as suggested in our preliminary data and from earlier research on the impact of the day hospital (further details avallable from authors upon request). The bulk of the costs associated with in-patient care are tied up in hotel costs and overheads, and most units run on much lower staffing ratios than we are able to offer in Home Options. This results in a paradox that in-patient units, despite having the most severely ill patients, often have the lowest staffing levels and the most inexperienced staff. Around $80 \%$ of new members of staff appointed to the Home Options Service have come from our in-patient unit, resulting in an exodus of experienced staff and potential problems of recruitment to the in-patient unit. Problems of this kind appear to be widespread, with a recent one-day survey of in-patient units across the UK reporting that $31 \%$ of nursing posts surveyed were filled by staff not working on permanent contracts (Ford et al, 1998).

Staff burn-out and low morale do not appear to be problems within the service at present, and staff turnover has been low. This seems to have been achieved through attention to supervision and training and an emphasis on multidisciplinary working. The service is demanding of doctors' time and the funding we received did not provide for any additional medical input. Although the service already had some dedicated 
medical time, it would certainly benefit from more medical input than is currently available.

It is also more difficult to monitor intensively the risk when treating patients at home, and although there is no research evidence that a service of this kind leads to more serious incidents, media attention to any disasters that occur in community-based services is likely to be more fierce. Staff working within such a service may therefore feel a greater burden of responsibility for the safety of patients and others. A detailed risk assessment tool has been helpful in addressing this area, and senior medical staff are always available to discuss concerns. The higher level of identified suicide risk among the Home Options patients may be partly attributable to a greater degree of vigilance among the Home Options staff and the use of a formalised screening tool that is not used routinely for in-patients. However, this finding is in keeping with our clinical impression that the Home Options Service is able to manage patients with significant suicide ideation, provided that they are able to cooperate with the team, and that patients requiring inpatient admission are much more likely to present with disturbed behaviour or aggression.

\section{Conclusions}

Innovative models of mental health service delivery are often criticised for their dependence on research funding and on the enthusiasm of a few individuals, leading to a lack of sustainability and generalisability. The incremental development of the day hospital service in Central Manchester is an example of an innovative service that has been able to build on its own strengths, learn from locally initiated research and maintain an impetus for change over more than a decade. Its location in an area with the highest level of need for mental health services in England (Smith et al, 1996) suggests that the model should be sustainable in other inner-city districts.

The model of service delivery and the management of the change process have been described here in some detail because visitors from other services tend to be particularly interested in these issues. Our preliminary evaluation data demonstrate continued differences between the inpatient population and those patients who can be treated successfully in the Home Options Service. The activity data suggest an increase in admission rates compared with the previous day hospital service, with a short overall length of stay. We are currently carrying out a more detailed evaluation to compare patients treated in the new service with those treated in the original day hospital, and also will be exploring the impact of the new service on in-patient admission rates.

\section{References}

Creed, F., Anthony, P.. Godbert, K., et al (1989) Treatment of severe psychiatric illness in a day hospital. British Journal of Psychiatry, 154, 341-347.

-. BLACK, D., ANTHONY, P., et al (1990) Randomised controlled trial of day patient versus in-patient psychiatric treatment. British Medical Journal, soo. 1033-1037.

-. MBaya. P., LANCASHiRE, S., et al (1997) Cost effectiveness of day and in-patient psychiatric treatment. British Medical Joumal, 314. 1381-1385.

FORD, R., DUNCAN, G.. WARNER. L., et al (1998) One-day survey by the Mental Health Act Commission of acute adult psychiatric in-patient wards in England and Wales. British Medical Journal. 317, 1279-1283.

Marks, I., ConNOLly, J.. MUWEN, M., et al (1994) Homebased versus hospital-based care for people with sertous mental illness. British Journal of Psychiatry. 185. 179-194.

Smrth, P., Sheldon, T. A. \& Martin, S. (1996) An index of need for psychiatric services based on in-patient utilisation. British Journal of Psychiatry, 169. 308-317.

WooD, H. (1994) What do service users want from mental health services? Report to the Audit Commission for Finding a Place: a Review of Mental Health Services for Adults. London: HMSO

WYKES, T. \& STURT, E. (1986) The measurement of socia behaviour in psychiatric patients: an assessment of the reliability and validity of the Social Behaviour Schedule. British Journal of Psychiatry. 148, 1-11.

WORLD HEALTH ORGANIZATION (1992) The ICD-10 Classification of Mental and Behavioural Disorders: Clinical Descriptions and Diagnostic Guidelines. Geneva: WHO.

-Judy Harrison, Consultant Psychiatrist and Amanda Poynton. Consultant Psychiatrist: John Marshall, Home Options Service Manager; Richard Gater, Senior Lecturer; and Francis Creed, Professor, Department of Psychiatry. Manchester Royal Infirmary, Oxford Road, Manchester M13 9WL

*Correspondence 